Quellung der Epithelzellen sowohl in den Lieberkühn'schen Drüsen, als in dem Oberflächenepithel. Die Becherzellen warev nicht nerklich vermehrt und nur spärliche Mitosen vorhanden. Das Darmstiick der zweiten Fistel war ganz normal, es fiel jedoch die sehr grosse Zahl der Mitosen im Epithel der Drüsenschläuche auf.

Zum Schluss sei es mir gestattet, Herrn Geleimrath Prof. Dr. Hermann für seine freundliche Anregung, sowie Herrn Prof. Dr. Langendorff für sein freundliches Entgegenkommen meinen wärmsten Dank auszusprechen.

(Aus dem physiologischen Institut zu Künigslerg i. Pr.)

\title{
Ueber graphische Darstellung der Actionsströme des Muskels.
}

\author{
Von \\ Dr. F. Matthias, Assistent.
}

(Hierzu 'Tafel III.)

Die Untersuchungen, über welche in den folgenden Blättern berichtet werden soll, sind nach einem Verfahren angestellt, dessen Principien von Herrn Geheimrath Hermann vor etwa einem Jahre in seiner Abhandlung "Ueber Rheotachygraphie" ${ }^{1}$ ) bereits auseinander gesetat worden sind. Die damals in Aussicht gestellte genauere Beschreibung des Apparats, welcher unterdess etwas vollkommner ansgeführt worden ist, soll hier zunächst gegeben werden.

Wie bekannt, ist der Apparat, dessen Benutzung mir von Herrn Geheimrath Hermann in liebenswiirdigster Weise überlassen wurde, im Wesentlichen nichts anderes als das Bernsteinsche Rheotom in der Form, die ihm von Hermann seiner Zeit gegeben wurde ${ }^{2}$, nur sind noch zur Bewerkstelligung einer con-

1) Dies Archiv Bd. 49, S. 539 ff.

2) Dies Archiv Bd. 31, S. 600, Taf. VIl. Das benutzte Rheotom ist von F. Meyer in Zürich, die Zuthaten vom hiesigen Mechaniker Wipprecht ausgeführt. 
tinuirlichen Verschiebung des Boussulcontacts eine Anzahl Aenderungen vorgenommen. Fig. 1, Tafel III stellt den Apparat von oben gesehen, Fig. 2 im Profil dar; in ein Drittel der natïrlichen Grösse.

Auf die untere Fläche der Ebonitscheibe ist ein Zahnkranz $K$ ans Messing aufgeschraubt und diese so in ein Zahnrad verwandelt. Der Fuss $F^{r}$ des Rlueotoms trägt einen mit zwei Schrauben befestigten, etwas nach oben gerichteten $\operatorname{Arm} A_{1}$, durch dessen Ende in verticaler Richtung die Axe des Triebes $T_{1}$ gesteckt ist; diese wird durch den Kopf einer von unten in sie eingelassenen Schraube in ihrem Achsloch gehalten. Der Trieb greift in den Zahnkranz $K$ ein; anf das obere Ende seiner Axe ist eine Schnurscheibe $S_{1}$ gesteckt unl mit 2 kleinen Schrauben befestigt. Diese Schnurscheibe kann mit einer grösseren $S_{2}$ oder dem Zahnrade $Z$ vertauscht werden. An Arm $A_{1}$ kann ein zweiter $A_{2}$ ebenfalls mit 2 schrauben befestigt werden, der wieder einen Trieb $T_{2}$ in das Zahnrad $Z$ eingreifend träigt. Anf diesem zweiten Trieb können wiederum die Schnurscheiben $S_{1}$ und $S_{2}$ nit kleinen Schrauben befestigt werden.

Contactrad und Ebonitscheibe des Rheotoms werden durch ein einfaches und bequemes, vom MecLaniker Herrn W ipp re eht nach den Angaben des Herrn Geheimrath Hermann construirtes Vorgelegre in Gang gesetzt. Der T-förmige ans Gusseisen bestehende Fuss a a ca desselben wird mit melreren Sehrauben, welche durch die mit ${ }^{c}$ in der Abbildung bezeichneten Lücher gesteckt werden, so auf dem Experimentirtisch befestigt, dass das freie Ende seines längeren Sehenkels ïber den Tischrand hinansrayt. Im Schnittpunkt der beiden Schenkel des Fusses erhebt sich eine Säule $b$ von $2 \mathrm{~cm}$ Dicke, deren oberer, etwas dinnerer Theil als Axe fur. eine hölzerne Stufenscheibe $c$ und ein gusseisernes Rad $d$, beide auf ein Messingfutter $c$ aufgesetzt, dient. Stufenscheibe und Rad sind mit Nuthen versehen, ein Gleiten derselben anf der Axe wird durch eine in letztere von oben eingelassene schraube mit breitem Kopf verhindert. Auf einer der drei Speichen des Rades ist ein Knopf 7i angebracht, welcher als Handhabe bei der Drehnng des Vorgeleges dient. Das Rad setzt durch eine dünne Lederpese y das $^{2}$ Contactrad des Rheotoms, der kleinste Schnurlauf der Stufenscheibe (durch Pese $p_{1}$ ) das Getriebe der Ebonitscheibe in Bewegung; die zwischen beiden liegenden Schnurläufe sind für den Fall angebracht, 
dass die Verwendung eines Motors zum Betriebe des Apparats gewählt werden sollte ${ }^{1}$ ).

Die Festlegung der gegenseitigen Stellung von Rheotom und Vorgelege ist folgendermaassen bewerkstelligt. Der lange Schenkel des T-förmigen Fusses ist an seinem freien Ende verbreitert und mit einem rechteckigen Schlitz versehen; in diesen ist ein cylindrisches Eisenstiuck $g$ eingesetzt, das nach oben in die Scheibe $f$ übergeht, das ganze wird durch eine starke Schraube $h$ mit Unterlegscheibe von unten her in dem Schlitz fixirt. Drei durch die Scheibe $f$ gehende Schrauben mit versenkten Köpfen greifen in die Eisenplatte des Rheotomfusses ein und drïcken diese gegen die Scheibe $f$. Mit dieser Einrichtung kann das Rheotom in jeder beliebigen Stellung fixirt und die Spannung der Lederpesen nach Bedarf variirt werden, es liegt darin für eine Reihe von Fällen eine grosse Bequemlichkeit. Soll zum Beispiel während des Versuchs von einer gewissen Verlangsamung zu einer andern übergegangen werden, so wird, nachdem die Pesen abgenommen und die entsprechenden Räder am Getriebe gewechselt sind, die Schraube $h$ gelockert, die wieder aufgelegten Pesen durch Drehung des Rheotoms um seine verticale Axe und Verschieben in dem Schlitz gespannt, und die Schraube angezogen. Soll ferner die untere Pese einmal gekreuzt, im nächsten Versuch ungekreuzt aufgelegt werden, wie es zur Aufnahme des Vorganges direet und in zeitli cher U m k h r an $\mathrm{g}^{2}$ ) nothwendig wird, so kann wieder dureli Drelsung des Rheotoms um die Schraube $h$ die Spannung der Pese ohne grossen Zeitverlust erfolgen.

Der eben beschriebene Apparat gestattet bei Benutzung einer oder beider Uebersetzungen und der Schnurscheibe $S_{1}$ und $S_{2}$ Verlangsamungen des Ablaufes des untersuchten Vorganges im Verhältniss von $1 \mathrm{zu} 230,347,1242$ und $2006^{3}$ ).

1) Bekanntlich ist die Drehgeschwindigkeit ohne Einfluss auf das Resultat, so dass die Drehung mit der Hand völlig genügt; vgl. Hermann, dies Archiv Bd. 49, S. 547.

2) Vgl. Bd. 49, S. 545 .

3) Die von Hermann a. a. 0. mitgetheilten Zahlen boziehen sich auf das damals benutzte hölzerne Vorgelege (vgl. a. a. O. S. 546). 
Versucheam Froschgastrocnemius.

Einem in bekaunter Weise mit seinem Nerven präparirten Froschgastrocnemius, der mit Nadeln anf einer Korkplatte ausgespannt ist, werden an der Achillessehne und in der Nähe des nervösen Aequators unpolarisirbare Röhrenelectroden der H e rman n'schen Modification ${ }^{1}$ ) angelegt. Muskel und Electroden befinden sich in einem Kreise, welcher ausser dem Boussolcontact des Rheotoms, einen geraden Compensator und die 4 Spiralen eines Spiegelgalvanometers mit äusserst leichtem aperiodischem Magneten $\left.{ }^{2}\right)$ enthält; in die Leitung zum Galvanometer ist ein $d u$ Boisscher Vorreiberschliussel als Nebenschliessung eingeschaltet. Dor Reizcontact ist mit 2 Daniell'schen Elementen in den primären Kreis cines Inductionsapparats eingeschaltet, dic secundäre Spirale des letzteren mit Vorreiberschliissel als Nebenschliessung ist zu zwei Zinkelectroden abgeleitet, auf welche der Nerv mit einem möglichst fern vom Muskd liegenden Theil aufgelegt wird. Das Präparat betindet sich mit seinen Electroden in einer fenchten Kammer.

In einer Anzahl von Versuchen, bei welchen die Magnetbewogungen mit Fernohr und Scala abgelesen wurden, zeigte es sich, dass für die phasischen Actionsstrime des Froschgastrocnemitus die Verlangsamung von 347 (an dem beschriebenen Apparat durch Aufsetzen der Schnurscheibe $S_{2}$ auf $T_{1}$ erreichbar) von den mir zu Gebote stehenden die geeignetste war. Bei geringerer Verlangsamung lagen die den Magneten treffenden einzelnen Impulse zu weit auseinander, un za ciner einheitlichen Wirkung: verschmolzen zu werden, bei grösserer wurde die Datuer des Versuchs so verlängert, dass der Muskel bereits vor Beendigung dessclben bei der zicmlich hohen Frequenz der Reizmng, 3-12 Reize pro Sccunde, vollstandig erschöpft war.

Nach längeren Bemiihungen gelang es mir, trotz der sich in unerwarteter Weise häufenden Schwierigkeiten brauchhare Beobachtungen arn Fernrobr zn machen. Bei Verwendung kräftiger, nicht ermiideter Muskeln machte der Magnet zuerst eine langsame riemlich ausgicbige Bewegung im sinne eines absteigenden Stromes, dann eine umgelsehrte weniger grosse, um schliesslich mit geringen Abweichungen zum Nullpunkt zurückzukehren.

1) Vgl. dies Archiv Bd. 42, s. 7 !?.

2) Vgl. cbendaselbst $\mathrm{S} .77$. 
Ich sclritt nun zur graphischen Registrirung der Magnetbewegungen und bediente mich dazu des von Hermann fiil seine phonophotographischen Untersuchungen ${ }^{1}$ ) erfundenen Verfahrens. Die zur Beleuchtung des verticalen Spalts dienende Siemens'sche Flachdecklampe stellte ich in möglichst grosser Entfernung von der Boussole auf, um etwaige Wirkungen ihrer regulirenden Electromagneten auf den Boussolmagneten auszuschliessen oder doch möglichst abzuschwächen. Andere, nicht electrische Lichtquellen ${ }^{2}$ ), mit denen ich zahlreiche Versuche anstellte, erwiesen sich theils als zu schwach, theils waren sie so schwierig in ihrer Handhabung und so unzuverlässig, dass sie die ohnehin recht complicirten Versuche fast bis zur Unausfuhrbarkeit erschwerten. Bei einer Entfernung von sechs Metern zwischen Boussole und Lampe habe ich niemals irgend welche Störungen wahrgenommen. Ferner fand ich für meine Versuche das weniger empfindliche Eastman'sche Bromsilberpapier vortheilhafter, als die hochempfindlichen Papiersorten von Dr. Stolze, die bei der langsamen Bewegung des zeichnenden Lichtpunktes nur schwer eine scharfe Zeichnung zu Staude kommen liessen.

Un die zur Entwerfung eines scharfen Bildes des beleuchteten Spalts nothwendigen Linsen möglichst nahe am Boussolspiegel in den Weg der Lichtstrahlen einzuschalten, nahm ich die den Spiegel vor Luftströmungen schïtzende Messingkappe ab und ersetate sie durch ein kleines cylindrisches Pappgebäuse, in dessen an einer Seite abgeplatteten Mantel die betreffenden Linsen eingekittet waren.

Zur Verzeichuugg von Zeitmarken auf den Curven wurde eine einfache Vorkehrung getroffen. Vor der electrischen Laterne. wurde ein Baltzar'scher Zeitmarkirer so angebracht, dass sein Schreibhebel mit einew von ihm getragenen, sehr leichten und mit Russ geschwärzten Glimmerfähnchen vertical vor den Spalt zu stehen kam und diesen gerade noch verdeckte, wenn der Anker nicht von dem Electromagneten angezogen war. Es wurde nun der Zeitmarkirer in einen Kreis eingeschaltet, der ausser einer Stromquelle eine Baltzar'sche Contactuhr enthielt. Bei jeder

1) Dies Archiv Bd. 15, S. $582 \mathrm{ff}$.

2) Gas- und Magnesiumlicht in Duboscq'scher Laterne, Auer'sches Glühlicht etc. 
von der Contactuhr bewirkten Oeffnung des Kreises verdeckte also das Glimmerfübnchen den Spalt, und die auf dem lichtempfindlichen Papier verzeichnete Curve erfuhr eine Unterbrechung. In meinen Versuchen stellte ich die Contactuhr stets so, dass alle zwei Secunden eine Unterbrechung eintrat.

Der Versuch gestaltete sich nun in folgender Weise. Nachdem das Bild des verticalen Spalts genau auf den Cylindermantel des auf mittlere Geschwindigkeit gestellten Kymographions und die markirte Mitte des horizontalen Spalts (s. oben S. 12) eingestellt, das Papier mit zwei Gummiringen auf den Cylinder gespannt und gegen Nebenlicht gehörig gesichert war, wurde das Präparat angefertigt, in die feuchte Kammer gebracht, und Reiz- und Ableitungselectroden angelegt. Während ich dann das Spaltbild im Ange behielt, schloss ein Assistent den Boussolcontact, riumte die Nebenschliessung zur Boussole weg und handhabte den Compensator nach meinem Commando zur Beseitigung von Ablenkungen durch etwa vorhandene Ruheströme. Hatte das Spaltbild seine Ruhestellung erreicht, so setate der Assistent mit der einen Hand das Rheotom in Bewegung und öfuete mit der andern die Nebenschliessung des Reizapparats; zugleich löste ich die Arretirung des Kynographionnhrwerks. Bei gelungenen Versuchen komnte ich stets eine liewegung des Spaltbildes zuerst in der dem absteigenden Strom, dann in der dem anfsteigenden entsprechenden Richtung wahrnehmen; die letztere war vermindert oder fehlte, weun der Muskel bereits längere Zeit grereizt war. D. h. es bestätigte sich einfach die Angabe $\mathrm{Her} m$ an $n$ n's ${ }^{1}$ ), welcher zuerst 1877 bei indirecter Reizung den doppelsinnigen Aetionsstrom beobachtete, unmittelbar darauf auch am menschlichen Muskel'²), und endlich auch am Nerven ${ }^{3}$ ). Kurz vorher hatte du Bois-Reymond auf das Bestimuteste behauptet, er habe ,niemals andere als absolut negative Schwankung gesehen" $\left.{ }^{\sharp}\right)$ (d.h. nur die crste Phase des Actionsstroms). Wie es kam, dass einem so hervorragenden Experimentator eine so prägnante Erscheinung gäuzlich entging, Lat $\mathrm{H}$ ermann $n^{\bar{x}}$ ) vollständig aufgeklärt.

1) Dies Archiv Bd. 16, s, wis tf.

2) Ebenda Bd. 1t, S. 410 ete.

3) Ebenda Bd. 24, s. 246 ete.

4) Arch. f. Anat. u. Plysiol. 1473. \$. 58\%.

5) A. a. 0. Ba. $16,5.243$. 
Der Versuch wurde nun so oft wiederholt, als die Grösse des aufgespannten Papierstreifens es gestattete, alsdann die Gasflamme, welche das im ïbrigen verdunkelte Zimmer schwach erleuchtete, ausgelöscht, und bei rothem Licht mit dem Eisenoxalatentwickler die Zeichnung hervorgerufen. Das erschienene Bild belehrte sofort tiber etwa nöthig gewordene Regulirangen der Licht. intensität, der Spaltbreite und iuber den Zustand des Präparats. Es ist mir bei der grossen Anzahl von Versuchen, die ieh im Laufe der Untersuchung angestellt habe, nie ein Muskel vorgekommen, der mehr als drei vollständige Curven geliefert bätte; in dem vierten Versuch fehlte die zweite Phase des Vorganges entweder ganz, oder war nur noch mit Mühe zu erkennen. Auf Tafel III ist in Nr. $1-5$ and 11 eine Auswahl gelungener Curven wiedergegeben; sie zeigen sämmtlich eine doppelsinnige Schwankung des Magneten entsprechend den beiden Phasen des Actionsstromes. Dass der Magnet dem verlangsamten Vorgange mit grosser Treue folgte, zeigen sebr schön die Curven Nr. 9 und 10. Sie sind beide unmittelbar nach einander an demselben Präparat aufgenommen, Nr. 9 bei natürlichem, Nr. 10 bei verkehrtem Ablauf des Vorgangs, d. h. bei umgekehrter Rotation der Ebonitscheibe. Auch hier bei $\mathrm{Nr} .10$ ist die zweite Phase vorhanden und die erste, die ihr in der Zeichnung folgt, die grössere, Umstände, welche jedenfalls den Verdacht ansschliessen, es könne sich hier um Einflüsse gehandelt haben, deren Ursache ausserhalb des Muskels lägen.

An sämmtlichen Curven fällt auf den ersten Blick der Unterschied der Grösse beider Phasen auf; der Grund dafür ist, wie schon dureh die angefïhrten Untersuchungen Hermann's über die Actionsströme des Muskels und Nerven bekannt ist, in einer Interferenz der Galvanometerwirkungen beider Phasen durch theilweise Superposition derselben zu suchen. Die Erregung hat eben die obere Ableitungsstelle noch nicht vollständig passirt, wenn sie an der unteren bereits anlangt. In den Curven 6-8 findet diese Thatsache eine weitere Bestätigung. Es fehlt, wie man sieht, in allen dreien die zweite Phase; bei ihrer Aufnahme, sie stammen der Reihe nach von denselben Präparaten, wie die Curven 1-3, war der Versuch an demselben Muskel hereits mehrmals wiederholt, sodass in Folge von Ermiidung die Erregung die nntere Ab. leitungsstelle nur noch mit sehr geringer Intensität erreichte und 
die der letzteren angehörende Phase des Actionsstromes nicht mehr zu Stande kam, oder der durch die Electroden abgeleitete Antheil derselben zil seliwach war, um auf die Boussole zu wirken. Die jetzt allein verzeichnete erste Phase zieht sich jedoch in allen drei Fïllen länger hin als in den Beispielen 1-3, auch ist ihre Amplitude grösser. Ich zeichnete nun eine Curre mit beiden Phasen und eine solche mit der ersten allein, die an demselben Präparat kurz nach einander aufgenommen waren, anf derselben Abscissenaxe ibereinander und construirte durch Ansmessen der Ordinaten die zweite Phase in ibrer wahren Form. Als Beispiele solcher Reductionen mögen die Curven 12 und 12a dienen. Es zeigt sich hier, dass die zweite Phase wohl etwas kleiner ist als die erste, was anf das Decrement der Erregung zu beziehen ist, im Wesentlichen sich aber von ibr nicht unterseheidet und dass die Form der Curve 1, welche der Reduction zu Grunde gelegt ist, wie vorher gesagt, durch das zeitliche Zusammenfallen der Phasen bedingt ist.

Um die Zulässigkeit des geschilderten Verfahrens zu priffen stellte ich auf den Rath des Herrn Gebeimrath Herm an n folgenden Versuch an. Bekanntlich kann man die zweite Phase durch Herstellung eines kïnstlichen Querschnitts am distalen Muskelende vollständig beseitigen ${ }^{1}$ ). Nachdem nun an einem Muskel der Vorgang einmal vollständig aufgenommen war, wurde die untere Ableitungsstelle mit Silbernitrat in Substanz geätzt; nach Compensation des Demarcationsstromes wurde dann der Versuch wiederbolt. Die gewonnenen, nunmehr einphasischen Curven wurden in derselben Weise wie Nr. 1 und 6 der Tafel III in Nr. 12 iibereinander gezeichnet und die zweite Phase construirt. Nr. 15 und 16 stammen von solchen Versuchen her. Sie zeigen in der Hauptsache dasselbe wie Nr. 12 und bestätigen nur die an dieser Curve gemachten Beobachtungen; es erscheint jedoch bei beiden das Maximum der zweiten Phase noch kleiner im Verbältniss zn dem der ersten, als dies bei Nr. 12 der Fall war. Es hat dies seinen Grund wieder in dem Decrement der Erregung, welches gerade bei den hier verwendeten Mnskeln besonders gross war, da die Frösche, mit denen gearbeitet wurde, schon lange Zeit in der Gefangenschaft und ohne Nahrung gelebt, ilhre Muskeln also an Widerstandsfähigkeit betrüchtlich eingebiisst hatten.

Aus dem zeitliehen Abstande beider Plıasen kann man die

1) Vergl. Hermann, dies Archir Bd. 16, s. 236 . 
Fortpflanzangsgeschwindigkeit der Erregung im Muskel zu ermitteln suchen, wie es zuerst Bernstein bei directer, Herman n am Menschen bei indirecter Reizung gethan hat. Aber es ist klar, dass es nicht richtig ist, den Zeitabstand der scheinbaren Maxima beider Phasen mit der Zeit $7 u$ identificiren, welche die Erregung braucht um die Strecke zwischen beiden Ableitungsstellen zu durchlaufen. Denn wegen der schon oben erwäbnten Interferenz ist das Maximum der zweiten Phase nicht blos vermindert, sondern anch zeitlich hinausgeschoben, wie Hermann bereits hervorgeboben hat (cit. oben S. 75). Aber durch das hier mitgetheilte Verfahren lïsst sich der wahre Verlauf des zweiten, abterminalen Actionsstroms leicht feststellen. Nach Aufzeichnung des doppelsinnigen Stromes wird die zweite Phase durch Aetzung beseitigt, und die nunmebr ungestörte erste, atterminale Phase für sich aufgeschrieben. Durch Auftragung der zweiten Curve auf die erste liisst sich durch Ordinatensubtraction die zweite Phase vollständig ermitteln. Man kann dies Verfahren die Separation beider Phasen nennen.

An einer Anzahl so reducirter Curven habe ich Bestimmungen der Fortpflanzungsgeschwindigkeit vorgenommen. Die absolute Zeit ist, wie oben (S. 75) angegeben, durch Unterbrechungen im Abstande von je 2 Secunden markirt, die Länge des abgeleiteten Muskelstiicks wurde nach jedem Versuch bestimmt. In der folgenden kleinen Tabelle sind einige Beispiele soleher Bestimmungen mitgetheilt.

\begin{tabular}{|c|c|c|c|c|c|c|}
\hline $\mathrm{Nr}$. & $\begin{array}{c}\text { der Zeit- } \\
\text { marken } \\
\text { mm }\end{array}$ & $\begin{array}{r}\text { Absta } \\
\text { der Cur } \\
\mathrm{mm}\end{array}$ & Secunden & \begin{tabular}{|c|} 
Länge \\
der \\
abgeleiteten \\
Muskelstrecke \\
mm \\
\end{tabular} & $\begin{array}{l}\text { Verlang- } \\
\text { samungs- } \\
\text { coëfficient }\end{array}$ & $\begin{array}{c}\text { Fortplan- } \\
\text { zungsgeschw. } \\
\text { der Erregung } \\
\mathrm{m}\end{array}$ \\
\hline 1. & 5,0 & 4,5 & 1,8 & 17 & 347 & 3,27 \\
\hline 2. & 5,0 & 3,0 & 1,2 & 22 & 347 & 6,36 \\
\hline 3. & 5,0 & 4,0 & 1,6 & 20 & 347 & 4,34 \\
\hline 4 & 7,5 & 7,5 & 2,0 & 25 & 347 & 4,33 \\
\hline
\end{tabular}

In dem Controllversuch $\mathrm{Nr} .15$ fällt sofort die lange Dauer der zweiten Phase auf. Die Erregung nimmt also nicht allein an Intensitït ab, sie pflanzt sich gegen das Faserende auch mit geringerer Geschwindigkeit fort, um so mehr, je grösser das Decre- 
ment ist. Es werden schon deshalb alle Bestimmungen der Fortpflanzungsgeschwindigkeit der Erregung an ausgeschnittenen Muskeln zu kleine Werthe ergeben, um so mehr zu klein, je grösser das Decrement.

Hieraus erklären sich die sehr verschiedenen Werthe der obigen Tabelle. Bei Nr. 2 war das Decrement sehr gering (siehe Nr. 12 a ґ. 'Taf. III) im Gegensatz zu Nr.1 (siehe Nr. 12 Taf. III).

Im Verlauf der Untersuchung erschien es, winnschenswerth, ausser der Zeit auch den Reizmoment auf der Curve zu verzeichnen, d. h. durch temporäre Spaltung diejenigen Schliessungen des Reizeontactes, welche auf die Schlusszeit des Bonssoleontactes fielen, auf der photographirten Linie durch Unterbreclungen zu markiren.

Zu diesem Zweck schaltete ish in den die Zeit markirenden Kreis den Contact eines polarisirten Telegraphenrelais, welches mir von der hiesigen Oberpostdirection bereitwilligst zur Verfügung gestellt wurde, ein. Die Klemmenpare von Reiz- und Boussolcontact wurden symmetrisch mit den seitlichen Klemmen zweier Pohlseher Wippen ohne Kreuz verbunden, deren Biigel so an eine Holzleiste gebunden waren, dass beide Wippen mit einem Griff umgelegt werden konnten. Von je zwei Klemmen der cinen Seite diser Doppelwippe wurde dann znm Reizapparat und Boussolkreise abgeleitet, sodass beide Kreise von einander isolirt geschlossen werden konnten. Von den vier Klenmen der andern Seite wurden zwei, verschiedenen Wippen angehörige, mit einander verbunden, die beiden noch übrigen führten zu einem Kreise, welcher ein Leclanché-Element und die magnetisirende Spirale des Relais enthielt. Wurde die Wippe nach dieser Seite gelegt, so befanden sich also Reiz- nnd Boussolcontact mit dem Relais in einem Kreise, bei gleichzeitigem Schluss beider Contacte wurde der Anker des Relais angezogen und der in den zeitmarkirenden Kreis eingeschaltete Contact desselben geöffnet, in diesem Moment liess der Baltzar'sche Zeitmarkirer seinen Anker los und das Glimmerfühnchen verdeckte für kurze Zeit den beleuchteten Spalt.

Der Versuch musste nun in der Weise modifieirt werden, dass nach vollendeter Compensation die Doppelwippe nach der Relaisscite gelegt, das Kymographion und dann das Rheotom in Gang gesetzt wurden. Z/ur Zeit der gleichzeitigen Contactschlüsse macht das Glimmerfihnchen zitternde Bewegungen, waren diese 
voriuber, so wurde die Wippe schnell umgelegt und der eigentliche Versuch begann. Die Nebenschliessung des Reizapparats blieb lier beständig geöffnet. Die Curven 2, 3, 6, 8 sind mit Markirung des Reizmoments aufgenommen, derselbe stellt sich als eine Reihe kurzer Unterbrechungen (auf den Curven mit $r$ bezeichnet) dar, dic um so länger ist, je grösser die Dauer der einzelnen Boussolschlïsse war. Dieses Verfahren kann zur Bestimmung eines Minimalwerthes der Fortpflanzungsgeschwindigkeit der Erregung im Nerven benutzt werden. Der Abstand zwischen Beginn des Reizmoments und der ersten Phase des Actionsstromes muss auf die Zeit verrechnet werden, welche die Erregung brauchte, um von der Reizstelle bis zum Muskel zu gelangen. Dieser Zeitwerth enthält aber zugleich die Latenzzeit des Nervenendorgans, er muss deshalb für die Berechnung der Fortpflanzungsgeschwindigkeit der Erregung im Nerven aus der Differenz der Zeiten und dem Abstande der Reizstelle vom nervösen Aequator nothwendig za gross und der erhaltene Werth $t$ zu klein werden, wie das aus einer Anzahl solcher Berechnungen gefundene Mittel 11,488 Meter beweist. Durch demnächst anzustellende graphische Versuche iiber die Actionsströme des Nerven hoffe ich anch für diese Grösse genaue Werthe festzustellen.

\section{Versucbeander Vorderarmmusculatur des Mensehen.}

Im Anfange meiner Untersuchungen botmir die von Herman n ${ }^{1}$ ) gemachte Erfahrung, dass der Vorderarm des lebenden Menschen für Versuche über die phasischen Actionsströme ein besonders günstiges Object ist, einen willkommenen Ausweg aus der Verlegenheit, die mir die Schwierigkeit der Versuche an sich und die Unzulänglichkeit des Materials (die vorhandenen Frösche waren bereits vor langer Zeit gefangen und frische wegen der vorgeriickten Jahreszeit schwer zu erhalten) bereiteten.

Die Versuche, die ich am menschlichen Vorderarm bei einer Verlangsamung des Vorganges von 1400 anstellte, sowohl mit Beobachtung am Fernrohr, als auch mit graphischer Darstellung, führten zu den ersten mit dem graphischen Verfahren erzielten Erfolgèn.

1) Dies Archiv Bd. 16, S. 410. 
Die Versuche wurden im Uebrigen in derselben Weise angestellt, wie die von Hermann a. a. 0 . mitgetheilten, nur wurde stets mit vollkommen aufgeschobener secundärer Spirale und fünf klcinen Grove'schen Elementen gereizt. Die Reizelectroden ferner wurden auf einem zur besseren Isolirung mit Paraffin gekochten breiten Kork befestigt und mit einem Riemen an Schulter oder Oberarm der Versuchsperson anbandagirt, je nachdem sie höber oder tiefer auf den Nerven aufgesetzt werden sollten. Es war so die Lage der Reizelectroden eine viel festere und sicherere, als wenn sie von der Versuchsperson mit einer Handhabe auf den Plexus brachialis gedriickt wurden.

Finige Beispiele am Fernrobr gemachter Beobachtungen sind in der untenstehenden Tabelle mitgetheilt. Nach einer Verschiebung der Scala in Sinne eines anfsteigenden Stromes, des Secretionsstromes, von wechselnder Grösse und Schnelligkeit, rückte diese 25 bis 50 Theilstriche nach der einem absteigenden Strom entsprechenden Seite, um dann iiber den Nullpunkt anuähernd ebenso weit nach der andern Seite zu gehen, schliesslich kehrte sie mit abuehmender Geschwindigkeit gegen den Nullpunkt zurïck.

\begin{tabular}{|c|c|c|c|c|}
\hline \multirow{2}{*}{$\mathrm{Nr}$. } & \multirow{2}{*}{ Yullpunkt } & \multirow{2}{*}{\multicolumn{2}{|c|}{$\begin{array}{l}\text { Maximum } \\
\text { del ab- der anf- } \\
\text { steigenden|steigenden }\end{array}$}} & \multirow{3}{*}{$\begin{array}{l}\begin{array}{c}\text { Nullpunkt } \\
\text { bei fort- } \\
\text { dauer'lder } \\
\text { Reizung }\end{array} \\
405\end{array}$} \\
\hline & & & & \\
\hline 1. & 415 & 455 & 375 & \\
\hline 2. & 476 & 515 & .40 & 475 \\
\hline 3. & 510 & 545 & 480 & 510 \\
\hline 4. & 525 & 560 & 500 & 520 \\
\hline 5. & 510 & 585 & 490 & 510 \\
\hline 6. & 510 & 537 & 492 & 510 \\
\hline 7. & 377 & 400 & 345 & 362 \\
\hline 8. & 371 & 405 & 143 & 363 \\
\hline 9. & 372 & 416 & 325 & 365 \\
\hline 10. & 455 & 475 & 435 & 455 \\
\hline
\end{tabular}

Der Umstand, dass in der Mebrzahl der Versuche der Nullpunkt nicht wieder erreicht wurde und die zweite Phase grösser erschien als die erste, ist durch die Einmischung des allmählich zu seiner vollen Intensität ansteigenden Secretionsstromes zu erklären. In den Versuchen 3,5,6 und 10, welche angestellt wurden, 
82 F. Matthias: Ueber graphische Darstell. der Actionsströme des Muskels.

indem schon einige Zeit vor Beginn der Ableitung der Actionsströme gereizt war, batte der Secretionsstrom schon in Anfange des eigentlichen Versuchs seine volle Höhe erreicht, so dass sein Ansteigen nicht melr störend eingreifen konnte.

Versuche mit graphischer Darstellung bestätigten die am Ferurohr gemachten Beobachtungen. Die Curven 13 und 14 mögen als Beispiele dienen. Bei Curve 13 begann die Reizung gleichzeitig mit dem eigentlichen Versuch, bei 14 schon längere Zeit vorber. Die geringe Grösse der Amplituden in diesen Curven erklärt sich aus der geringen Entfernung des Kymographioncylinders von dem Boussolspiegel und der damit verbundenen geringen Vergrösserung der Magnetbewegungen. Es fehlen an diesen Curven noch die Markirung des Reizmoments und der Zeit, Dinge, zn deren Ausführung ich erst im Verlauf der Untersuchungen an Froschmuskeln gelangte. Bei einer Wiederholung der Versuche am Menschen, die ich in nächster Zeit vorzunehmen gedenke, soll dies nachgeholt werden; ich hoffe dann vollkommenere Curven zu liefern, die auch zur Bestimmung der Fortpflanzungsgeschwindigkeit der Erregung im Musksel mehr geeignet sind als die vorliegenden; Bestimmungen der letzteren, die ich auf Grund einer grösseren Anzahl solcher Curven vornahm, ergaben Werthe von 10,7 bis $12,7 \mathrm{~m}$ pro Secunde, fast genan übereinstimmend mit den Ergebnissen Hermann's. 